\title{
Analytical solutions for fermions on a thick brane with a piecewise and smooth warp factor
}

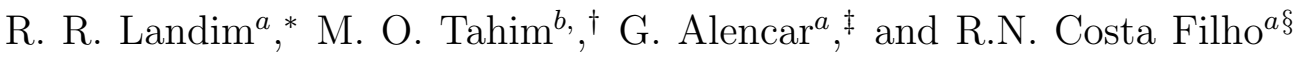 \\ ${ }^{a}$ Departamento de Fúsica, Universidade Federal do Ceará, \\ 60451-970 Fortaleza, Ceará, Brazil and \\ ${ }^{b}$ Universidade Estadual do Ceará, Faculdade de Educação, \\ Ciências e Letras do Sertão Central - R. Epitácio Pessoa, \\ 2554, 63.900-000 Quixadá, Ceará, Brazil.
}

(Dated: October 3, 2018)

\begin{abstract}
In this paper we study analytical solutions for fermion localization in Randall-Sundrum (RS) models. We show that there exist special couplings between scalar fields and fermions giving us discrete massive localizable modes. Besides this we obtain resonances in some models by analytical methods.
\end{abstract}




\section{INTRODUCTION}

The study of gravity resonances in Randall-Sundrum models [1, 2] is of great importance because, since stable states for most fields do not exist, it points to the possibility of finding unstable massive modes in the membrane. Much of these studies used smooth versions of the background with the RS warp factor recovered for large $z[3$ [10]. The emergence of resonances is an interesting phenomenological possibility that has been approached mainly numerically. However, some examples have been studied where an integrable solution was found for bosonic fields [11, 12]. In order to obtain these solutions a specific form of the background was chosen such that a simple potential was obtained for an associated Schrödinger equation. That leads to the question: what kind of background allows analytical solutions? The answer to this question was given in [13] where the authors found an associated equation leading to the correct background. That was possible by the introduction of an arbitrary function describing the region near the (thick) brane. This arbitrary function makes the connection with the effective potential of the Schrödinger like equation. With this, the backgrounds used in the literature [11, 12, 14] are just particular solutions of this equation. Besides, many others solutions were found. In particular, the fermion case was studied in ref. [14] but the solutions were found only numerically. It happens that the fermion case introduces additional complications because of the coupling with the scalar field. Anyway, it is possible to circumvent this and generalize the idea used for the gravity case: here we find a few examples of analytical solutions. Therefore, the complete structure of resonances associated with each of these new solutions are also obtained. The way chosen to describe the background of the thick brane gives us a better understanding of its structure and can lead to new possibilities.

This letter is organized as follows: in the first section we give a brief review of spin- $1 / 2$ fermion background. In the second section we describe fermion in a profile with piecewise warp factor. The third section is devoted to study fermion in a background with smooth warp factor. Finally, we present the conclusions and perspectives. 


\section{THE FERMION SETUP}

We regard here the problem of studying fermions in a Randall-Sundrum like scenario. Usually it is considered the coupling between fermions and the gravitational fields alongside a coupling between fermions and a scalar field $\phi$, the stuff the membrane is made of. Indeed, the membrane is regarded as a domain wall embedded in $D=5$. Studies of fermion localization in this context reveals a very natural mechanism of producing chiral fields in $D=4$ [4 6, 15 24]. The new ingredient here is that, if the warp factor is defined in a piecewise way, so must be the scalar field $\phi$. The usual coupling is the Yukawa one that will be generalized to allow a more complete analysis.

First, we give a brief review of the fermion background. Let us consider a single scalar field thick brane $\phi$ with a potential $V(\phi)$ in $(4+1)$-dimensional curved space-time with cosmological constant $\Lambda$. The action describing this model is given by

$$
S=\int d^{5} x \sqrt{-g}\left[\frac{1}{2 \kappa_{5}^{2}}(R-2 \Lambda)-\frac{1}{2} g^{M N} \partial_{M} \phi \partial_{N} \phi-V(\phi)\right],
$$

where $R$ is the scalar curvature, $g=\operatorname{det}\left[g_{M N}\right], M, N=0,1,2,3,4$, and $\kappa_{5}^{2}=8 \pi G_{5}$ with $G_{5}$ the five-dimensional Newtonian gravitational constant. We will use the metric in the conformal coordinates and the line-element is assumed to be

$$
d s^{2}=g_{M N} d x^{M} d x^{N}=e^{2 A(z)}\left(\eta_{\mu \nu} d x^{\mu} d x^{\nu}+d z^{2}\right),
$$

where $e^{2 A(z)}$ is the warp factor. The metric tensor $\eta_{\mu \nu}$ presents signature in the form $(-,+,+,+,+)$, and $z$ stands for the coordinate of the extra spatial dimension. The scalar

field is considered to be a function of $z$ only: $\phi=\phi(z)$. From the metric ansatz (2) the Einstein tensor is given by

$$
\begin{aligned}
& G_{\mu \nu}=\left(3 A^{\prime 2}+3 A^{\prime \prime}\right) \eta_{\mu \nu}, \quad G_{44}=6 A^{\prime 2} \\
& G_{4 \mu}=0
\end{aligned}
$$

where the prime denotes derivative with respect to the $z$ coordinate.

The Euler-Lagrange equations for the action (1) with the metric ansatz (2) after some manipulations are given by:

$$
\begin{aligned}
\kappa_{5}^{2} \phi^{\prime 2} & =3\left(A^{2}-A^{\prime \prime}\right) \\
V(\phi) & =-\frac{1}{2 \kappa_{5}^{2}}\left(9 A^{\prime 2}+3 A^{\prime \prime}\right) e^{-2 A}-\frac{\Lambda}{\kappa_{5}} \\
\frac{d V(\phi)}{d \phi} & =\left(3 A^{\prime} \phi^{\prime}+\phi^{\prime \prime}\right) e^{-2 A} .
\end{aligned}
$$


If the $Z_{2}$ symmetry is imposed, namely $A(z)=A(-z)$, we can see from Eqs. (5) and (6) that in the limit $|z| \rightarrow \infty$ with $\phi^{\prime}(z) \rightarrow 0$ and $V(\phi) \rightarrow 0$ we have $A(z) \rightarrow-\ln \left(k_{0}|z|+\beta\right)$ and $\Lambda=-6 k_{0}^{2}$. In this case the bulk is asymptotically AdS. It is worthwhile to mention that the condition $\lim _{|z| \rightarrow \infty} \phi^{\prime}(z) \rightarrow 0$ can be satisfied by kink solution $\left(\lim _{z \rightarrow \pm \infty} \phi(z)= \pm c_{0}, c_{0}>0\right)$. We shall restrict the bulk asymptotically AdS and kink solutions (with $\phi(z)=-\phi(-z)$ ) through this paper. We also make $\kappa_{5}=1$ for simplicity.

In this background, the Dirac fermionic action for a spin 1/2 field coupled to the scalar field $\phi$ is

$$
S_{1 / 2}=\int d^{5} x \sqrt{-g}\left[\Psi \Gamma^{M} D_{M} \Psi-\eta \bar{\Psi} F(\phi) \Psi\right]
$$

where $D_{M}=\partial_{M}+\omega_{M}, \omega_{M}$ being the spin connection. The $\Gamma^{M}=e_{N}{ }^{M} \gamma^{N}$ are the Dirac matrices in the five dimensional curved space-time and $e_{N}^{M}$ are the vielbeins: $e_{A}{ }^{M} e_{B}{ }^{N} \eta^{A B}=$ $g^{M N}$.

The Dirac equation in five-dimensional space-time can be derived from the action(8)

$$
\left[\gamma^{\mu} \partial_{\mu}+\gamma^{5}\left(\partial_{z}+2 \partial_{z} A\right)-\eta e^{A} F(\phi)\right] \Psi(x, z)=0
$$

where $\gamma^{\mu} \partial_{\mu}$ is the four-dimensional Dirac operator on the brane.

Due to the presence of $\gamma^{5}$ in the Eq. (9), is convenient to make the chiral decomposition of the fermions in Kaluza-Klein (KK) modes[14]:

$$
\Psi(x, z)=e^{-2 A} \sum_{n}\left[\psi_{\mathrm{L} n}(x) \psi_{-}^{n}(z)+\psi_{\mathrm{R} n}(x) \psi_{+}^{n}(z)\right]
$$

where $\psi_{\mathrm{L} n}(x)=-\gamma^{5} \psi_{\mathrm{L} n}(x)$ and $\psi_{\mathrm{R} n}(x)=\gamma^{5} \psi_{\mathrm{R} n}(x)$ are the left-handed and right-handed components of the Dirac fermion fields on the brane, respectively. They satisfy the four-dimensional massive Dirac equations in the form of $\gamma^{\mu} \partial_{\mu} \psi_{\mathrm{L} n}(x)=m_{n} \psi_{\mathrm{R} n}(x)$ and $\gamma^{\mu} \partial_{\mu} \psi_{\mathrm{R} n}(x)=m_{n} \psi_{\mathrm{L} n}(x)$. The KK modes $\psi_{ \pm}^{n}(z)$ obeys the coupled equations

$$
\frac{d \psi_{ \pm}}{d z} \mp \eta e^{A(z)} F(\phi(z)) \psi_{ \pm}=\mp m \psi_{\mp} .
$$

From these we obtain the Schrödinger like equation for each mode

$$
-\psi_{ \pm}^{\prime \prime}(z)+U_{ \pm}(z) \psi(z)_{ \pm}=m^{2} \psi_{ \pm}(z)
$$


where the effective potential $U(z)$ depends on the warp factor as bellow

$$
U_{ \pm}(z)=\left(\eta F(\phi(z)) e^{A(z)}\right)^{2} \pm \frac{d}{d z}\left(\eta F(\phi(z)) e^{A(z)}\right) .
$$

The study of the massive modes must be done more carefully. This happens because when the potential in (12) has the asymptotic behaviour $\lim _{|z| \rightarrow \infty} U_{ \pm}(z)=0, \psi_{ \pm}(z)$ are linear combination of plane waves and therefore not normalizable.

For massive modes, like in non-relativistic quantum mechanics, we will use the transmission coefficient to study possible resonances [9]. Usually, the transmission coefficient represents the probability flux of the transmitted wave relative to the incident wave. In our case, the transmission coefficient represents the probability of a fermionic massive mode to pass through the brane. The idea of the existence of a resonant mode is that for a given mass the transmission coefficient has a peak at this mass value. In our study of resonances we limit the solutions of $(12)$ that have asymptotic planes wave form.

\section{THE PIECEWISE CASE}

In this section the warp factor $A(z)$ will be defined in two regions, $|z| \leq d$ and $|z| \geq d$ :

$$
A(z)= \begin{cases}-\ln (f(z)), & |z| \leq d ; \\ -\ln \left[\left(k_{0}(|z|+\beta)\right],\right. & |z| \geq d,\end{cases}
$$

where $d$, beta are positive constants and $f(z)$ is called associated function with $f(z)>0$ in $|z| \leq d, k_{0}=\sqrt{-\Lambda / 6}$. This is enough to establish how the membrane curves the spacetime with the bulk being asymptotically $\operatorname{AdS}[11,12$.

In order to get the desired smooth versions, the functions $A, A^{\prime}$ and $A^{\prime \prime}$ must be continuous to give $\phi$ and $\phi^{\prime}$ continuous at $|z|=d$. That restricts the form of $f(z)$ in the membrane. For the gravitational field the choice of [11] was $A(z)=\frac{2}{3} \ln \cos \left(\sqrt{V_{0}}|z|\right)$ giving the effective potential $U=-V_{0}$ for $|z| \leq d$. That allows a detailed analytical study of the model. Recently, this result was found as a particular one belonging to a wider class of new exact solutions for the gravitational field [13]. The purpose here is to present a new way to generalize this idea in order to obtain analytical solutions for the fermion field as well.

We focus our attention in right fermions. The analysis is similar for left fermions. In order to implement the boundary conditions, we restrict to even functions $f(z)$ in $(14)$, 
guaranteeing that the boundary conditions are satisfied in both edges of the brane, $z=d$ and $z=-d$. The condition $A(0)=0$ implies that $f(0)=1$. Since $f(z)$ is an even function we also have $A^{\prime}(0)=0$. Using the equations (5) and (6) we obtain

$$
\phi^{\prime}(z)^{2}= \begin{cases}3 \frac{f^{\prime \prime}(z)}{f(z)}, & |z| \leq d, \\ 0, & |z| \geq d,\end{cases}
$$

and

$$
V(\phi(z))=\frac{3}{2} f^{\prime \prime}(z) f(z)-6 f^{\prime}(z)^{2}+6 k_{0}^{2} .
$$

From Eq. (11) we can see that the massless right modes obey the condition

$$
\frac{f_{0}^{\prime}(z)}{f_{0}(z)}=\eta e^{A(z)} F(\phi(z)) .
$$

With this condition, the potential of the schrodinger-like equation becomes

$$
U(z)=\frac{f_{0}^{\prime \prime}(z)}{f_{0}(z)} .
$$

If we choose $f_{0}(z)$ such that $(12)$ is analytically solved we can obtain analytically the resonances.

In order to implement the boundary conditions, we are restricted to even functions $f_{0}(z)=$ $f_{0}(-z)$ with $U(z)=U(-z)$ in $(18)$. This guarantees that the boundary condition in $z=d$ is satisfied also in $z=-d$. Our method consist in finding $U(z)$ and $F(\phi(z))$ that have analytical solution for Eq. (12). As we can see from (17), $F(\phi(z))$ is an odd function in $z$. From Eq. 15], $\phi(z)=c_{0}$ for $z \geq d$. We also have two regions for $f_{0}(z)$ :

$$
f_{0}(z)= \begin{cases}g_{0}(z), & |z| \leq d, \\ (|z|+\beta)^{\gamma}, & |z| \geq d,\end{cases}
$$

where $\gamma=\eta F\left(c_{0}\right) / k_{0}$ and $g_{0}(z)$ being an even function. In order to obtain a localizable zero mode, $\gamma<-1 / 2$. Now the potential in 18 becomes

$$
U(z)= \begin{cases}\frac{g_{0}^{\prime \prime}(z)}{g_{0}(z)}, & |z| \leq d, \\ \frac{\gamma(\gamma-1)}{(|z|+\beta)^{2}}, & |z| \geq d .\end{cases}
$$


The solution of $(12)$ for $|z| \geq d$ is a linear combination of Bessel functions of order $|\gamma|+1 / 2$. With the above considerations and by imposing continuity of $A(z), A^{\prime}(z), A^{\prime \prime}(z), f_{0}(z)$ and $f_{0}^{\prime}(z)$ at $z= \pm d$ we obtain

$$
\begin{array}{r}
f(d)=k_{0}(d+\beta), \\
f^{\prime}(d)=k_{0}, \\
f^{\prime \prime}(d)=0, \\
g_{0}(d)=(d+\beta)^{\gamma}, \\
g_{0}^{\prime}(d)=\gamma(d+\beta)^{\gamma-1},
\end{array}
$$

with the conditions $f(d)>0$.

The solution for right fermion of 12 will be given by

$$
\psi_{m}(z)= \begin{cases}E_{m}(z)+C_{m} F_{m}(z), & z \leq-d, \\ A_{m} g_{m}(z)+B_{m} h_{m}(z), & |z| \leq d, \\ D_{m} F_{m}(z), & z \geq d,\end{cases}
$$

where $E_{m}(z)=\sqrt{\frac{\pi u}{2}} H_{\nu}^{(2)}(u), F_{m}(z)=\sqrt{\frac{\pi u}{2}} H_{\nu}^{(1)}(u)$, with $u=m(|z|+\beta), \nu=(|\gamma|+1 / 2)$ and $g_{m}(z)\left(h_{m}(z)\right)$ are the even(odd) functions. The functions $E_{m}(z)$ and $F_{m}(z)$ have asymptotic behaviour $e^{i m z}$. In order to analyze the resonances, we fixed the coefficient of the incoming wave to one.

Using the fact that $g_{m}(z), E_{m}(z), F_{m}(z)$ are even and $h_{m}(z)$ are odd and the continuity of the $\psi_{m}(z), \psi_{m}^{\prime}(z)$ at $|z|=d$ we finally obtain the transmission coefficient

$$
T(m)=\frac{m^{2}\left|W\left(g_{m}, h_{m}\right)(d)\right|^{2}}{\left|W\left(F_{m}, g_{m}\right)(d) W\left(F_{m}, h_{m}\right)(d)\right|^{2}},
$$

where $W(f, g)(z)=f(z) g^{\prime}(z)-f^{\prime}(z) g(z)$ is the Wronskian. As applications of the method, consider the scalar field in $|z| \leq d$ given by $\phi(z)=a \sqrt{3} \sin (z)$. From Eq. 15 we obtain

$$
-f^{\prime \prime}(z)+a^{2} \cos ^{2}(z) f(z)=0 .
$$

The even solution of (28) is given by the Mathieu cosine functions [25]:

$$
f(z)=C\left(-\frac{a^{2}}{2}, \frac{a^{2}}{4}, z\right),
$$




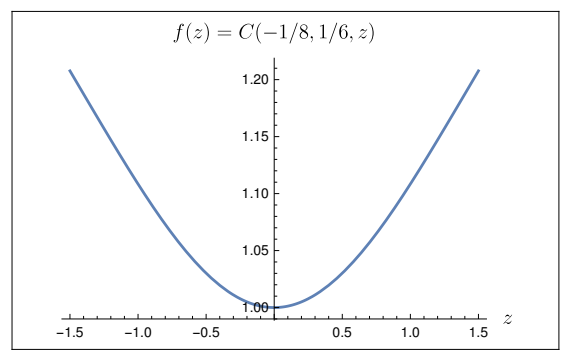

FIG. 1. The Mathieu cosine function $C(-1 / 8,1 / 16, z)$.

with $f(z)$ real and $f(0)=1$. From Eqs. (21), (22) and (23), we obtain $d=\pi / 2, k_{0}=0.65447$, $\beta=1.02593$ for $a=1 / 2$. The scalar potential can be written as

$$
\begin{aligned}
V(\phi)= & \frac{3}{8}\left(1-\frac{4 \phi^{2}}{3}\right) C^{2}\left(-\frac{1}{8}, \frac{1}{16}, \arcsin (2 \phi / \sqrt{3})\right) \\
& -6 C^{\prime 2}\left(-\frac{1}{8}, \frac{1}{16}, \arcsin (2 \phi / \sqrt{3}),\right)+6 k_{0}^{2}
\end{aligned}
$$

where $C^{\prime}(n, m, x)$ is the derivative of $C(n, m, x)$ with respect do $x$. The function $C(a, b, x)$ is obtained from the real part of MathieuC $(a, b, x)$ divided by the real part of MathieuC $(a, b, 0)$, where MathieuC $(a, b, x)$ is defined in the Mathematica software. For completeness, we show the plot of $f(z)=C(-1 / 8,1 / 16, z)$ in Fig. 1 .

For $g_{0}(z)=A_{0} \cos \left(\sqrt{V_{0}} z\right), V_{0}>0$ in $(19)$ we obtain $U(z)=-V_{0},|z| \leq \pi / 2$. From Eqs. (24) and 25) we have

$$
\begin{aligned}
& \gamma=-\left(\frac{\pi}{2}+\beta\right) \sqrt{V_{0}} \tan \left(\sqrt{V_{0}} \frac{\pi}{2}\right) \\
& A_{0}=\left(\frac{\pi}{2}+\beta\right)^{\gamma} / \cos \left(\sqrt{V_{0}} \frac{\pi}{2}\right) .
\end{aligned}
$$

Since $\gamma<-1 / 2$, we have some restrictions for the values of $V_{0}$. We also have that $\cos \left(\sqrt{V_{0}} \frac{\pi}{2}\right) \neq 0$ and $\sin \left(\sqrt{V_{0}} \frac{\pi}{2}\right) \neq 0$. This implies that $\sqrt{V_{0}}$ cannot be an integer. For $V_{0}=1 / 4$ we have $\gamma=-1.29836$ and $A_{0}=0.409675$. We show in Fig. 2, the exponential of two times the warp factor and the massless right fermion wave function for $V_{0}=1 / 4$.

Now using the equation (17), we show below the scalar fermion coupling

$$
\eta F(\phi)=\frac{-2 A_{0}}{\sqrt{3}+\sqrt{3-4 \phi^{2}}} C\left(-\frac{1}{8}, \frac{1}{16}, \arcsin (2 \phi / \sqrt{3})\right) .
$$

The solution of 12 for $|z| \leq d$ is a linear combination of $g_{m}(z)=\cos \left(\sqrt{m^{2}+V_{0}} z\right)$ and $h_{m}(z)=\sin \left(\sqrt{m^{2}+V_{0}} z\right)$. Now using the Eq. (27), we show in Fig. 3 the transmission coefficient for the right fermion. As physical result, we have a massive resonant mode near $m=0.9$. 


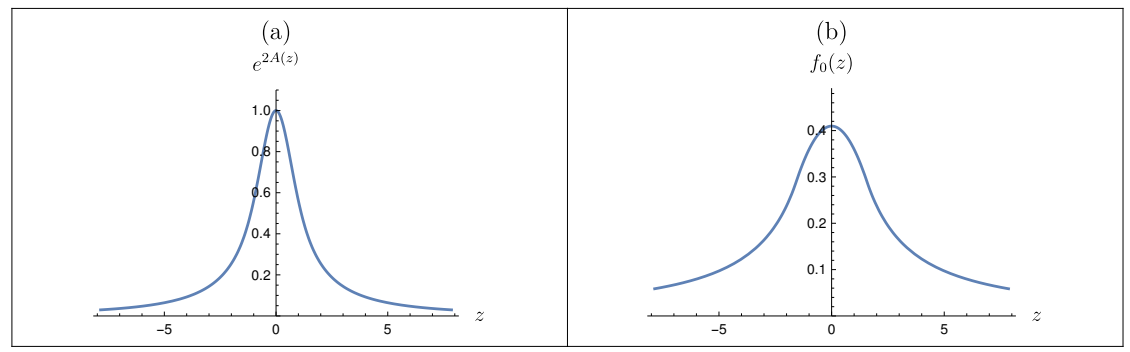

FIG. 2. (a) The exponential of two times the warp factor. (b) The zero mode of right fermion for $a=1 / 2$ and $V_{0}=1 / 4$.

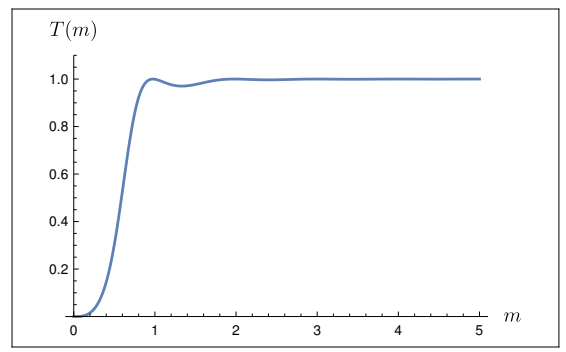

FIG. 3. The transmission coefficient of right fermion for $a=1 / 2$ and $V_{0}=1 / 4$.

\section{THE SMOOTH CASE}

In this section we consider a smooth warp factor given by

$$
A(z)=-\frac{1}{2} \ln \left(k_{0}^{2} z^{2}+1\right), \quad-6 k_{0}^{2}=\Lambda
$$

This type of warp factor was used in [26] to obtain resonances for Kalb-Ramond fields. From Eqs. (5) and (6) we obtain

$$
\begin{aligned}
\phi(z) & =\sqrt{3} \arctan \left(k_{0} z\right), \\
V(\phi) & =\frac{15 k_{0}^{2}}{2} \cos ^{2}\left(\frac{\phi}{\sqrt{3}}\right) .
\end{aligned}
$$

We consider for now, the Sine-Gordon-like fermion coupling: $F(\phi)=\sin (\phi / \sqrt{3})$. With this we obtain the potential

$$
U(z)=\frac{k_{0} \eta\left(-k_{0}^{2} z^{2}+k_{0} \eta z^{2}+1\right)}{\left(k_{0}^{2} z^{2}+1\right)^{2}} .
$$


Making the substitution $\psi(z)=\Phi(z)\left(1+k_{0}^{2} z^{2}\right)^{\eta / 2 a}$ and $x=-k_{0}^{2} z^{2}$ in 12 we arrive at the confluent Heun equation [27]

$$
x(x-1) w^{\prime \prime}(x)+\left(\left(\frac{1}{2}+\frac{\eta}{k_{0}}\right) x-\frac{1}{2}\right) w^{\prime}(x)+\frac{m^{2}}{4 k_{0}^{2}}(1-x) w(x)=0,
$$

where $\Phi(z)=w(x)$. The right massless mode $f_{0}(z)=C_{0}\left(1+k_{0}^{2} z^{2}\right)^{\eta / 2 k_{0}}$, is normalized for $\eta / k_{0}<-1 / 2$. The solution of (12) for massive right fermion is

$$
\psi(z)=\left(A H_{1}\left(-k_{0}^{2} z^{2}\right)+B H_{2}\left(-k_{0}^{2} z^{2}\right)\right)\left(1+k_{0}^{2} z^{2}\right)^{\eta / 2 k_{0}}
$$

where $H_{1}(x), H_{2}(x)$ are the confluent Heun functions [27] and $A, B$ are constants. In this case, we show the transmission coefficient in Fig, 4 for $k_{0}=1$ and $\eta=-1$. With these parameters we do not have massive resonant modes.

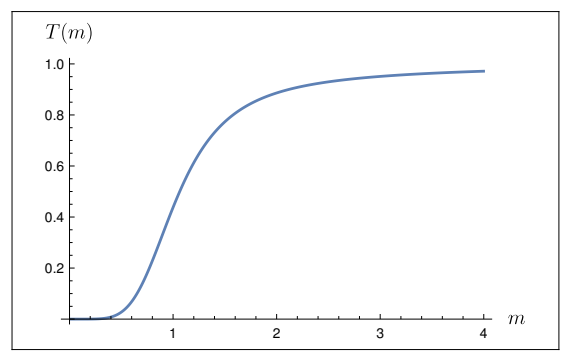

FIG. 4. The transmission coefficient of right fermion for Sine-Gordon coupling, with $k_{0}=1, \eta=-1$.

\section{Discrete massive modes}

Looking at the expression of the potential, given by Eq. (13), we can study what type of $F(\phi)$ coupling gives discrete massive modes. Asymptotically, for any warp factor we must have $A(z) \sim 1 / z$. In order to get bounded states, $U(z)$ must go to a constant or to infinity. In the case of $U(z)$ asymptotically going to a constant, we must have that $F(\phi) \sim z$. For the warp factor given by Eq. (34) and $F(\phi)=\tan (\phi / \sqrt{3})=k_{0} z$, the right potential is

$$
U(z)=\frac{k_{0} \eta}{\left(1+k_{0}^{2} z^{2}\right)^{3 / 2}}+\frac{k_{0}^{2} \eta^{2} z^{2}}{1+k_{0}^{2} z^{2}} .
$$

In this case, the right massless mode is given by $f_{0}(z)=C_{0} e^{\frac{\eta}{k_{0}} \sqrt{1+k_{0}^{2} z^{2}}}$ and is normalized for $\eta<0$. For the potential in Fig 5 , we obtain discrete massive modes $m=2.16,2.67,2.87$ for $k_{0}=1$ and $\eta=-3$. 


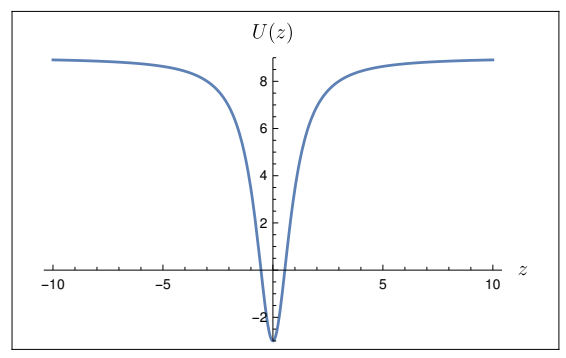

FIG. 5. The right potential profile for $F(\phi)=\tan (\phi / \sqrt{3})$, with $k_{0}=1, \eta=-3$.

It can be shown that is possible to obtain analytically discrete localizable modes. As mentioned before, the massless right mode obey $\eta e^{A} F(\phi)=f_{0}^{\prime} / f_{0}$, then the right and left potential reads

$$
\begin{aligned}
& U_{+}=\frac{f_{0}^{\prime \prime}}{f_{0}}, \\
& U_{-}=2\left(\frac{f_{0}^{\prime}}{f_{0}}\right)^{2}-\frac{f_{0}^{\prime \prime}}{f_{0}} .
\end{aligned}
$$

From the arbitrariness of $F(\phi)$, we can choose $f_{0}(z)$ in order to obtain normalizable modes for $\psi_{+}$and $\psi_{-}$. Let us consider the case where $U_{+}-U_{-}=\gamma$, with $\gamma$ constant. Then we obtain the solution

$$
\begin{array}{r}
f_{0}(z)=C e^{\frac{\gamma}{4} z^{2}} \\
U_{ \pm}(z)=\frac{\gamma^{2}}{4} z^{2} \pm \frac{\gamma}{2}
\end{array}
$$

Since $f_{0}(z)$ is the zero mode for right fermion, the localization occurs only if $\gamma<0$. In this case, taking $\gamma=-2 \omega^{2}$, we obtain the schrodinder-like equation

$$
-\psi_{ \pm}^{\prime \prime}(z)+\left(\omega^{4} z^{2} \mp \omega^{2}\right) \psi(z)_{ \pm}=m^{2} \psi_{ \pm}(z)
$$

This is the exactly the Schrödinger equation for the one dimensional harmonic oscillator. The massive modes are localizable only if $m=\sqrt{2 n} \omega$, for right fermions and $m=\sqrt{2 n+2} \omega$, for left fermions, $n=0,1,2 \cdots$. This gives the coupling

$$
\eta F(\phi)=-\frac{\omega^{2}}{k_{0}} \frac{\sin (\phi / \sqrt{3})}{\cos ^{2}(\phi \sqrt{3})} .
$$

This type of coupling was studied in another context in [28] for a symmetric $d S$ branes. This coupling provides analytical treatment and, in this particular case, gives a remarkable 
characteristic: localizable massive modes. It is then important to discuss this coupling in order to find some physical interpretation, if it exists in fact. Despite this, massive fermionic modes can be found in the context of inflating baby-skyrmion brane models [29, 30] and in other more usual scenarios [31].

\section{CONCLUSIONS AND PERSPECTIVES}

Here we have shown that it is possible to generalize the formalism to obtain analytical solutions to models of localizations of fermions in RS scenarios. This work is a generalization of previous results only applied to bosonic fields, namely, the gravitational, vector and scalar fields. The main idea is to make use of a warp factor defined in a piecewise way and solve an associated equation that describes its profile centered at the origin. With the analytical solution at hand it is quite easy to obtain and study the resonances in the models. We analyzed the conditions of fermion-scalar coupling which give localizable massive modes. For a very specific case we found an analytical coupling between fermions and the scalar field which gives localizable discrete massive modes. For a very specific case we found an analytical coupling between fermions and the scalar field which gives localizable discrete massive modes. These results are very interesting since the mass spectrum of particles is discrete. We believe that, despite the fact we are studying fermions, this formalism could open a new way to approach open problems as analytical study of non-abelian gauge field in branes[32]. More interesting yet is the discussion of models containing another kind of spinors, a result which can be applied to supersymmetric models. All of these questions are left for future works.

\section{ACKNOWLEDGMENTS}

We acknowledge the financial support provided by Fundação Cearense de Apoio ao Desenvolvimento Científico e Tecnológico (FUNCAP), the Conselho Nacional de Desenvolvimento Científico e Tecnológico (CNPq) and FUNCAP/CNPq/PRONEX.

* rrlandim@gmail.com 
$\dagger$ makarius.tahim@gmail.com

$\ddagger$ geova@fisica.ufc.br

$\S$ rai@fisica.ufc.br

[1] L. Randall and R. Sundrum, Phys.Rev.Lett. 83, 4690 (1999), arXiv:hep-th/9906064 [hep-th],

[2] L. Randall and R. Sundrum, Phys.Rev.Lett. 83, 3370 (1999), arXiv:hep-ph/9905221 [hep-ph].

[3] D. Bazeia and L. Losano, Phys.Rev. D73, 025016 (2006), arXiv:hep-th/0511193 [hep-th].

[4] Y.-X. Liu, J. Yang, Z.-H. Zhao, C.-E. Fu, and Y.-S. Duan, Phys.Rev. D80, 065019 (2009), arXiv:0904.1785 [hep-th],

[5] Z.-H. Zhao, Y.-X. Liu, and H.-T. Li, Class.Quant.Grav. 27, 185001 (2010), arXiv:0911.2572 [hep-th].

[6] J. Liang and Y.-S. Duan, Phys.Lett. B681, 172 (2009).

[7] G. Alencar, R. Landim, M. Tahim, C. Muniz, and R. Costa Filho, Phys.Lett. B693, 503 (2010), arXiv:1008.0678 [hep-th].

[8] R. Landim, G. Alencar, M. Tahim, M. Gomes, and R. Costa Filho, Europhys.Lett. 97, 20003 (2012), arXiv:1010.1548 [hep-th].

[9] R. Landim, G. Alencar, M. Tahim, and R. Costa Filho, JHEP 1108, 071 (2011), arXiv:1105.5573 [hep-th].

[10] R. Landim, G. Alencar, M. Tahim, and R. Costa Filho, JHEP 1202, 073 (2012), arXiv:1110.5855 [hep-th].

[11] M. Cvetic and M. Robnik, Phys.Rev. D77, 124003 (2008), arXiv:0801.0801 [hep-th],

[12] G. Alencar, R. Landim, M. Tahim, and R. C. Filho, JHEP 1301, 050 (2013), arXiv:1207.3054 [hep-th].

[13] R. Landim, G. Alencar, M. Tahim, and R. Costa Filho, Phys.Lett. B731, 131 (2014), arXiv:1310.2147 [hep-th].

[14] H.-T. Li, Y.-X. Liu, Z.-H. Zhao, and H. Guo, Phys.Rev. D83, 045006 (2011), arXiv:1006.4240 [hep-th].

[15] A. Kehagias and K. Tamvakis, Phys.Lett. B504, 38 (2001), arXiv:hep-th/0010112 [hep-th].

[16] V. A. Rubakov and M. E. Shaposhnikov, Phys. Lett. B125, 136 (1983).

[17] Y.-X. Liu, Z.-G. Xu, F.-W. Chen, and S.-W. Wei, Phys. Rev. D89, 086001 (2014), arXiv:1312.4145 [hep-th]. 
[18] A. Chumbes, A. Vasquez, and M. Hott, Phys.Rev. D83, 105010 (2011), arXiv:1012.1480 [hep-th].

[19] L. B. Castro and L. A. Meza, Europhys. Lett. 102, 21001 (2013), arXiv:1011.5872 [hep-th],

[20] R. Correa, A. de Souza Dutra, and M. Hott, Class.Quant.Grav. 28, 155012 (2011), arXiv:1011.1849 [hep-th],

[21] L. Castro, Phys.Rev. D83, 045002 (2011), arXiv:1008.3665 [hep-th]

[22] O. Castillo-Felisola and I. Schmidt, Phys. Rev. D82, 124062 (2010), arXiv:1008.1281 [hep-th],

[23] Y.-X. Liu, C.-E. Fu, L. Zhao, and Y.-S. Duan, Phys. Rev. D80, 065020 (2009), arXiv:0907.0910 [hep-th].

[24] A. Melfo, N. Pantoja, and J. D. Tempo, Phys. Rev. D73, 044033 (2006), arXiv:hepth/0601161 [hep-th],

[25] N. McLachlan, Theory and Applications of Mathieu Functions (Oxford, 1951).

[26] Y.-Z. Du, L. Zhao, Y. Zhong, C.-E. Fu, and H. Guo, Phys.Rev. D88, 024009 (2013), arXiv:1301.3204 [hep-th].

[27] L. J. El-Jaick and B. D. B. Figueiredo, J. Math. Phys. 49, 083508 (2008), arXiv:mathph/0807.2219 [math-ph],

[28] Y.-X. Liu, Z.-H. Zhao, S.-W. Wei, and Y.-S. Duan, JCAP 0902, 003 (2009), arXiv:0901.0782 [hep-th].

[29] T. Delsate and N. Sawado, Phys. Rev. D85, 065025 (2012), arXiv:1112.2714 [gr-qc],

[30] Y. Kodama, K. Kokubu, and N. Sawado, Phys. Rev. D79, 065024 (2009), arXiv:0812.2638 [hep-th].

[31] C. Ringeval, P. Peter, and J.-P. Uzan, Phys. Rev. D65, 044016 (2002), arXiv:hep-th/0109194 [hep-th].

[32] G. Alencar, R. R. Landim, C. R. Muniz, and R. N. Costa Filho, Phys. Rev. D92, 066006 (2015), arXiv:1502.02998 [hep-th]. 